\title{
Centroidal Momentum Matrix of a Humanoid Robot: Structure and Properties
}

\author{
David E. Orin and Ambarish Goswami
}

\begin{abstract}
The centroidal momentum of a humanoid robot is the sum of the individual link momenta, after projecting each to the robot's Center of Mass (CoM). Centroidal momentum is a linear function of the robot's generalized velocities and the Centroidal Momentum Matrix is the matrix form of this function. This matrix has been called both a Jacobian matrix and an inertia matrix by others. We show that it is actually a product of a Jacobian and an inertia matrix.

We establish the relationship between the Centroidal Momentum Matrix and the well-known joint-space inertia matrix. We present a Transformation Diagram that graphically captures the inter-relationships of the matrix operators and motion and momentum variables in Joint Space, CoM Space as well as the System Space.

The Centroidal Momentum Matrix is a local scaling function that maps the joint rates to the centroidal momentum. Following the concept of the manipulability ellipsoid, we propose the centroidal momentum ellipsoid that quantifies the momentum generation ability of the robot. We present a simulation plot showing the evolution of the singular values of the Centroidal Momentum Matrix during the walking motion of a humanoid.
\end{abstract}

Index Terms-momentum matrix, inertia matrix, angular momentum, linear momentum, matrix properties, centroidal momentum ellipsoid.

\section{Motivation}

The use of linear and angular momenta in humanoid robot control has been the topic of a number of recent research activities. Robot momenta is increasingly being explored as an important quantity in gait control [9], [10], [12], [14], [18], [19], [21], [22] as well as an indicator of balance [1], [7]. Yet, there is surprisingly little study on the nature of momenta in the context of humanoid robots which are floating-base, multibody dynamics systems ${ }^{1}$. In this paper we study the structure and properties of linear and angular momenta of humanoid robots using spatial notation for the dynamics [4], [6].

The spatial momentum vector of a humanoid robot consists of its linear and angular momenta. While the quantity of a robot's linear momentum is an absolute property and is inherently defined at its CoM, the angular momentum depends on the reference point at which it is computed. The aggregate angular momentum of a robot is obtained by summing up the angular momenta contributed by the

D. E. Orin is with the Department of Electrical and Computer Engineering, The Ohio State University, Columbus, OH 43210, U.S.A., email: orindece.osu.edu

A. Goswami is with the Honda Research Institute, Mountain View, CA 94041, U.S.A., email: agoswami@honda-ri.com

${ }^{1}$ Only recently we have seen a comprehensive study of angular momentum during human gait [8]. individual link segments. The centroidal angular momentum of a robot is the aggregate angular momentum referred to its Center of Mass (CoM) or centroid. The $6 \times 1$ centroidal momentum vector $\boldsymbol{h}_{G}$, which consists of the linear and centroidal angular momenta of the robot, is related to its $n \times 1$ joint velocity vector $\dot{\boldsymbol{q}}$ as:

$$
\boldsymbol{h}_{G}=\boldsymbol{A}_{G}(\boldsymbol{q}) \dot{\boldsymbol{q}} .
$$

The $6 \times n$ matrix $\boldsymbol{A}_{G}$ is called the Centroidal Momentum Matrix and is the focus of this paper. We study its properties, structure and relationships to other quantities. In our formulation, $\boldsymbol{A}_{G}(\boldsymbol{q})$ contains contributions both from the intersegmental joint variables of the robot as well as from the fictitious joint connecting the inertial frame and the floating base (detailed in Section II) of the humanoid. Note that $\boldsymbol{A}_{G}(\boldsymbol{q})$ is identical to the large matrix in the RHS of Eq. 1 of [10], with only the linear and angular parts interchanged.

The literature contains only a rare mention of matrices that map joint rates into aggregate momenta of a multibody dynamic system. In [3] the linear momentum Jacobian is computed as an intermediate step towards computing what the authors refer to as a force Jacobian. This formulation is used for animating articulated figures and does not contain angular momentum. In [15] the "angular momentum Jacobian" matrix is used to control the flight phase of a hopping robot. Finally, for resolved momentum control of humanoid robots, use has been made of "matrices which indicate how the joint speeds affect the linear momentum and angular momentum" [10]. Although they are called "inertia matrices" in this work, these matrices are identical to the "momentum Jacobian" matrices mentioned before.

Is $\boldsymbol{A}_{G}(\boldsymbol{q})$ an inertia matrix or a Jacobian matrix? Through a Transformation Diagram and through computation we will show that $\boldsymbol{A}_{G}(\boldsymbol{q})$ is the product of a pure inertia matrix and a pure Jacobian matrix.

From Eq. 1 we can think of $\boldsymbol{A}_{G}(\boldsymbol{q})$ as a local scaling function that maps the joint rates to the centroidal momentum. Following the concept of the manipulability ellipsoid [25], we will propose the centroidal momentum ellipsoid that quantifies the manner in which the joint rates are mapped to the centroidal momentum. Specific circumstances of gait and balance may impose specific requirements on the centroidal momentum and a study of the ellipsoid may provide information on the momentum limits in different directions of the robot as well as the best ways to achieve them.

The organization of this paper is as follows. First the velocity and momentum equations for a humanoid robot are derived using spatial notation. This is followed by a 
description of the structure and properties of the Centroidal Momentum Matrix. A Transformation Diagram containing the inter-relationships among motion and momentum variables is next presented. Then, the centroidal momentum ellipsoid is defined and a brief example given, followed by conclusions and a description of future work.

\section{Humanoid Robot Model}

In order to develop the dynamic model of a humanoid robot, the approach taken in [6] for rigid-body systems will be used. Spatial notation [4], [6] is a concise vector notation for describing rigid-body velocity, acceleration, inertia, etc., using $6 \mathrm{D}$ vectors and tensors, and is an integral part of the approach.

A humanoid can be modeled as a set of $N+1$ links interconnected by $N$ joints, of up to six degrees of freedom each, forming a tree-structure topology. The motion of the links are referenced to a fixed base (inertial frame) which is labeled 0 while the links are labeled from 1 through $N$. Numbering of the links may be done in any manner such that link $i$ 's predecessor toward the root (link 0), indicated by $p(i)$, is always less than $i$. Joints in the tree are numbered such that joint $i$ connects link $i$ to link $p(i)$. A coordinate frame is attached to each link to provide a reference for quantities associated with the link.

The relationship between connected links in the tree structure is described using the general joint model of Roberson and Schwertassek [23]. An $n_{i} \times 1$ vector $\dot{\boldsymbol{q}}_{i}$ relates the velocity of link $i$ to the velocity of its predecessor, link $p(i)$, where $n_{i}$ is the number of degrees of freedom at the joint connecting the two links. The free modes of the joint are represented by the $6 \times n_{i}$ matrix $\boldsymbol{\Phi}_{i}$, such that the spatial velocity of link $i$ is given as follows:

$$
\mathbf{v}_{i}=\left[\begin{array}{c}
\boldsymbol{\omega}_{i} \\
\boldsymbol{v}_{i}
\end{array}\right]={ }^{i} \boldsymbol{X}_{p(i)} \mathbf{v}_{p(i)}+\boldsymbol{\Phi}_{i} \dot{\boldsymbol{q}}_{i}
$$

where $\boldsymbol{\omega}_{i}$ and $\boldsymbol{v}_{i}$ are the angular and linear velocities of link $i$, respectively, as referenced to the link coordinate frame. ${ }^{i} \boldsymbol{X}_{p(i)}$ is a $6 \times 6$ spatial transform which transforms spatial motion vectors from $p(i)$ to $i$ coordinates. The matrix $\boldsymbol{\Phi}_{i}$ depends on the type of joint [23], [6]. It has full column rank, as does the orthogonal matrix $\boldsymbol{\Phi}_{i}^{c}$ representing the constrained modes of the joint, such that $\left[\boldsymbol{\Phi}_{i} \boldsymbol{\Phi}_{i}^{c}\right]$ is a basis of $\mathbb{R}^{6}$ and is invertible.

In order to model a humanoid when in flight, one of the links is modeled as a floating base (typically the torso) and numbered as link 1. A fictitious six degree-of-freedom (DoF) joint is inserted between the floating base and fixed base. In this case, $\boldsymbol{\Phi}_{1}=\mathbf{1}_{6 \times 6}$ where $\mathbf{1}_{6 \times 6}$ is the identity matrix. The Denavit-Hartenberg convention is used for single DoF

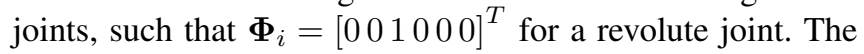
total number of degrees of freedom in the humanoid is $n$ where $n=\sum n_{i}$. Note that $n$ includes the six degrees of freedom for the floating base.

The spatial transform ${ }^{i} \boldsymbol{X}_{p(i)}$ may be composed from the position vector ${ }^{p(i)} \boldsymbol{p}_{i}$ from the origin of coordinate frame $p(i)$ to the origin of $i$, and the $3 \times 3$ rotation matrix ${ }^{i} \boldsymbol{R}_{p(i)}$ which transforms 3D vectors from coordinate frame $p(i)$ to $i$ :

$$
{ }^{i} \boldsymbol{X}_{p(i)}=\left[\begin{array}{cc}
{ }^{i} \boldsymbol{R}_{p(i)} & \mathbf{0} \\
{ }^{i} \boldsymbol{R}_{p(i)} \boldsymbol{S}\left({ }^{p(i)} \boldsymbol{p}_{i}\right)^{T} & { }^{i} \boldsymbol{R}_{p(i)}
\end{array}\right] .
$$

The quantity $\boldsymbol{S}(\boldsymbol{p})$ is the skew-symmetric matrix that satisfies $\boldsymbol{S}(\boldsymbol{p}) \boldsymbol{\omega}=\boldsymbol{p} \times \boldsymbol{\omega}$ for any 3D vector $\boldsymbol{\omega}$. It is defined by the equation

$$
\boldsymbol{S}(\boldsymbol{p})=\left[\begin{array}{ccc}
0 & -p_{z} & p_{y} \\
p_{z} & 0 & -p_{x} \\
-p_{y} & p_{x} & 0
\end{array}\right] .
$$

\section{A. Spatial Momentum}

The spatial momentum of each link may be computed from the spatial velocity as follows (see Fig. 1):

$$
\boldsymbol{h}_{i}=\left[\begin{array}{c}
\boldsymbol{k}_{i} \\
\boldsymbol{l}_{i}
\end{array}\right]=\boldsymbol{I}_{i} \mathbf{v}_{i},
$$

where $\boldsymbol{k}_{i}$ is the angular momentum, $\boldsymbol{l}_{i}$ is the linear momentum, and $\boldsymbol{I}_{i}$ is the spatial inertia for link $i$. The spatial inertia may be composed from the mass $m_{i}$, position vector to the center of mass $(\mathrm{CoM}) \boldsymbol{c}_{i}$, and $3 \times 3$ rotational inertia $\overline{\boldsymbol{I}}_{i}$, all relative to coordinate frame $i$ :

$$
\boldsymbol{I}_{i}=\left[\begin{array}{cc}
\overline{\boldsymbol{I}}_{i} & m_{i} \boldsymbol{S}\left(\boldsymbol{c}_{i}\right) \\
m_{i} \boldsymbol{S}\left(\boldsymbol{c}_{i}\right)^{T} & m_{i} \mathbf{1}
\end{array}\right]
$$

where

$$
\overline{\boldsymbol{I}}_{i}=\overline{\boldsymbol{I}}_{i}^{c m}+m_{i} \boldsymbol{S}\left(\boldsymbol{c}_{i}\right) \boldsymbol{S}\left(\boldsymbol{c}_{i}\right)^{T},
$$

and $\overline{\boldsymbol{I}}_{i}^{c m}$ is the rotational inertia about the CoM. Recall that if the origin of coordinate frame $i$ is chosen at the CoM, the off-diagonal blocks $m_{i} \boldsymbol{S}\left(\boldsymbol{c}_{i}\right)$ reduce to zero. If, in addition, the axes of coordinate frame $i$ are oriented along the principal axes of inertia, $\overline{\boldsymbol{I}}_{i}$ becomes a $3 \times 3$ diagonal matrix and $\boldsymbol{I}_{i}$ a $6 \times 6$ diagonal matrix.

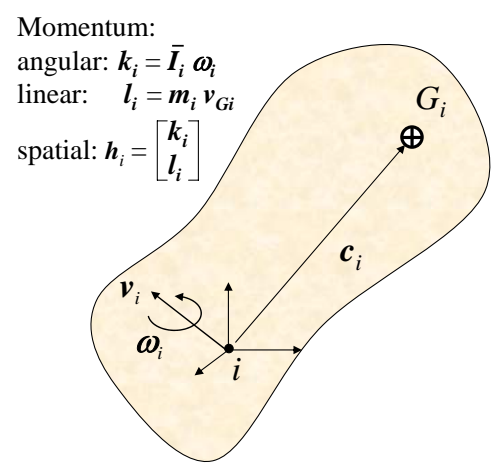

Fig. 1. Schematic depiction of a single rigid body: spatial momentum contains the angular and linear momenta.

\section{B. Global Notation}

It is possible to combine the equations for the velocity or momentum for all the links into a global set of equations [5]. To do so, composite vectors and matrices are defined, and these were the starting point of the spatial operator algebra developed by Rodriguez et al. [24]. Global notation 
is useful in developing a system Jacobian which leads to an expression for the Centroidal Momentum Matrix.

The difference between the velocity of a link and that of its predecessor may be determined by rearranging Eq. 2:

$$
\mathbf{v}_{i}-{ }^{i} \boldsymbol{X}_{p(i)} \mathbf{v}_{p(i)}=\boldsymbol{\Phi}_{i} \dot{\boldsymbol{q}}_{i}
$$

The relationship between all of the link velocities in the system $\mathbf{v}$ and the joint rates $\dot{\boldsymbol{q}}$ may be expressed through the use of an incidence matrix ${ }^{2} \boldsymbol{P}$ :

$$
\boldsymbol{P}_{\mathbf{v}}=\boldsymbol{\Phi} \dot{q}
$$

where

$$
\boldsymbol{P}_{i j}=\left\{\begin{array}{cl}
\mathbf{1}_{6 \times 6} & : j=i \\
-{ }^{i} \boldsymbol{X}_{p(i)} & : j=p(i) \\
\mathbf{0}_{6 \times 6} & : \text { otherwise }
\end{array}\right.
$$

and

$$
\begin{gathered}
\mathbf{v}=\left[\mathbf{v}_{1}^{T}, \mathbf{v}_{2}^{T}, \cdots \mathbf{v}_{i}^{T}, \cdots \mathbf{v}_{N}^{T}\right]^{T} \\
\dot{\boldsymbol{q}}=\left[\dot{\boldsymbol{q}}_{1}^{T}, \dot{\boldsymbol{q}}_{2}^{T}, \cdots \dot{\boldsymbol{q}}_{i}^{T}, \cdots \dot{\boldsymbol{q}}_{N}^{T}\right]^{T} \\
\mathbf{\Phi}=\operatorname{diag}\left[\boldsymbol{\Phi}_{1}, \boldsymbol{\Phi}_{2}, \cdots \boldsymbol{\Phi}_{i}, \cdots \boldsymbol{\Phi}_{N}\right] .
\end{gathered}
$$

Matrix $\boldsymbol{P}$ is sparse, lower-triangular, and easily inverted. Solving for $\mathbf{v}$ in Eq. 9 results in:

$$
\mathbf{v}=\boldsymbol{P}^{-1} \boldsymbol{\Phi} \dot{\boldsymbol{q}}=\boldsymbol{J} \dot{\boldsymbol{q}}
$$

where the system Jacobian $\boldsymbol{J}$ is defined to give the relationship between the system velocity and the joint rates $^{3}$ :

$$
\boldsymbol{J}=\boldsymbol{P}^{-1} \boldsymbol{\Phi}
$$

The elements of the system Jacobian are just the Jacobians for each of the links:

$$
\boldsymbol{J}=\left[\boldsymbol{J}_{1}^{T}, \boldsymbol{J}_{2}^{T}, \cdots \boldsymbol{J}_{i}^{T}, \cdots \boldsymbol{J}_{N}^{T}\right]^{T} .
$$

The momenta of all the links in the system may be determined as the product of the system velocity vector $\mathbf{v}$ and the system inertia $\boldsymbol{I}$; gathering all:

$$
\boldsymbol{h}=\boldsymbol{I} \mathbf{v},
$$

where $\boldsymbol{h}$ is the $6 N \times 1$ system momentum vector:

$$
\boldsymbol{h}=\left[\boldsymbol{h}_{1}^{T}, \boldsymbol{h}_{2}^{T}, \cdots \boldsymbol{h}_{i}^{T}, \cdots \boldsymbol{h}_{N}^{T}\right]^{T}
$$

and the $6 N \times 6 N$ system inertia matrix is defined as:

$$
\boldsymbol{I}=\operatorname{diag}\left[\boldsymbol{I}_{1}, \boldsymbol{I}_{2}, \cdots \boldsymbol{I}_{i}, \cdots \boldsymbol{I}_{N}\right] .
$$

\footnotetext{
${ }^{2}$ The elements of a standard incidence matrix are the scalars $+1,-1$, and 0 , not $6 \times 6$ matrices. The incidence matrix here also includes the spatial transform between link coordinates.

${ }^{3}$ The system Jacobian is not to be confused with the manipulator Jacobian in traditional fixed-based manipulators. The system Jacobian is an extension of the manipulator Jacobian and can contain it as one of its blocks if the corresponding coordinate frame is located at the task point.
}

\section{STRUCTURE ANd PRoperties of Centroidal Momentum Matrix}

\section{A. Formulation of Centroidal Momentum Matrix}

As shown in Eq. 1, the Centroidal Momentum Matrix gives the relationship between the joint rates and centroidal momentum. In order to find the relationship between this matrix and the link inertias and Jacobians, the concept of the system momentum matrix $\boldsymbol{A}$ is first presented. The system momentum matrix $\boldsymbol{A}$ gives the relationship between the system momentum vector and the joint rates: $\boldsymbol{h}=\boldsymbol{A} \dot{\boldsymbol{q}}$.

Substituting the expression for the system velocity in Eq. 13 into Eq. 16, gives:

$$
\boldsymbol{h}=\boldsymbol{I} \boldsymbol{J} \dot{\boldsymbol{q}} .
$$

From this, and using the definition of the system momentum matrix, we can write:

$$
\boldsymbol{A}=\boldsymbol{I} \boldsymbol{J} .
$$

The system momentum matrix is just the product of the system inertia matrix and the system Jacobian. It includes the momentum matrix for each link and is of size $6 N \times n$ :

$$
\boldsymbol{A}=\left[\boldsymbol{A}_{1}^{T}, \boldsymbol{A}_{2}^{T}, \cdots \boldsymbol{A}_{i}^{T}, \cdots \boldsymbol{A}_{N}^{T}\right]^{T},
$$

with

$$
\boldsymbol{A}_{i}=\boldsymbol{I}_{i} \boldsymbol{J}_{i}
$$

As defined, the spatial momentum of each link $\boldsymbol{h}_{i}$ is most naturally expressed in its own coordinate system. As a measure of dynamic stability or for control, it is useful to combine the momenta for the links by projecting the momenta to a common coordinate frame. A convenient frame is one set at the instantaneous CoM or the centroid of the system $G$, and whose coordinate axes are parallel to those of the inertial coordinate frame 0 . Noting that the spatial momentum may be projected as any other force-type vector [6], the following equation may be used to calculate the spatial momentum at the CoM of the system (see Fig. 2):

$$
\boldsymbol{h}_{G}=\sum_{i=1}^{N}{ }^{i} \boldsymbol{X}_{G}^{T} \boldsymbol{h}_{i}=\sum_{i=1}^{N}{ }^{i} \boldsymbol{X}_{G}^{T} \boldsymbol{A}_{i} \dot{\boldsymbol{q}} .
$$

The centroidal momentum may be expressed as a function of the system momentum:

$$
\boldsymbol{h}_{G}=\mathbf{X}_{G}^{T} \boldsymbol{h},
$$

where $\mathbf{X}_{G}$ is defined as the projection matrix, for motion vectors, from centroidal coordinates to link coordinates and is given as follows:

$$
\mathbf{X}_{G}=\left[{ }^{1} \boldsymbol{X}_{G}^{T},{ }^{2} \boldsymbol{X}_{G}^{T}, \cdots{ }^{i} \boldsymbol{X}_{G}^{T}, \cdots{ }^{N} \boldsymbol{X}_{G}^{T}\right]^{T} .
$$

The centroidal momentum may also be expressed as a function of the system momentum matrix $\boldsymbol{A}$ :

$$
\boldsymbol{h}_{G}=\mathbf{X}_{G}^{T} \boldsymbol{A} \dot{\boldsymbol{q}} \text {. }
$$

The Centroidal Momentum Matrix, $\boldsymbol{A}_{G}$, may then be defined as:

$$
\boldsymbol{A}_{G}=\mathbf{X}_{G}^{T} \boldsymbol{A}
$$




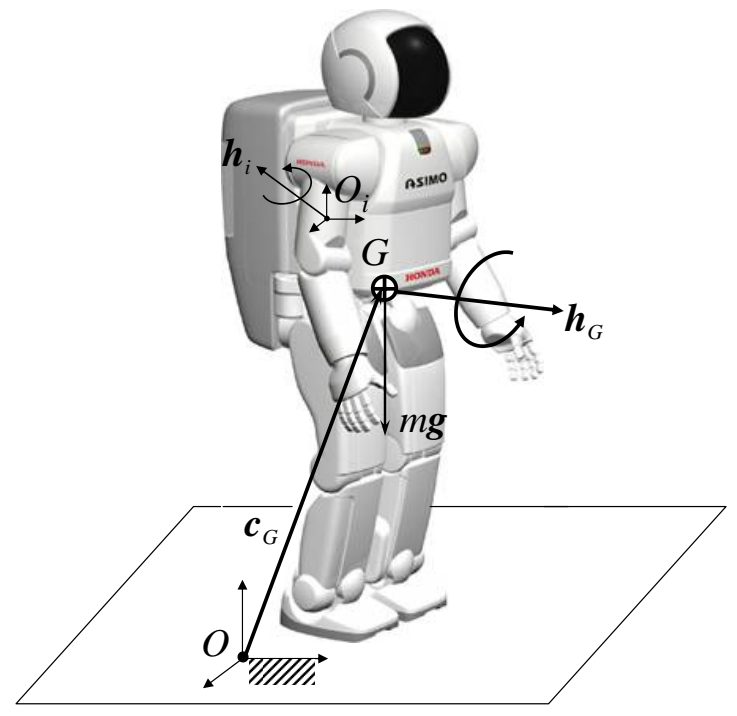

Fig. 2. Humanoid robot showing link and centroidal momentum vectors. The inertial frame is located at $O$ and the robot CoM is given by $\boldsymbol{c}_{G}$. The reference frame of link $i$ is located at $O_{i}$. The centroidal momentum $\boldsymbol{h}_{G}$ can be obtained from either Eq. 24 or Eq. 28.

so that it gives the relationship between the centroidal momentum and the joint rates:

$$
\boldsymbol{h}_{G}=\boldsymbol{A}_{G} \dot{\boldsymbol{q}}
$$

\section{B. Introduction to Transformation Diagram}

In order to establish the relationships between the Centroidal Momentum Matrix and other matrices, we have developed a graphical representation called the Transformation Diagram. The Transformation Diagram, shown in Fig. 3, captures the inter-relationships between the vector variables and transformation matrices related to the Centroidal Momentum Matrix. In doing so it reveals unexplored transformations between these quantities that can provide key computational tools and enhanced physical insight. The Transformation Diagram relates the velocity and momentum variables in joint space, system space and CoM space.

The $n$-dimensional joint space ${ }^{4}$ represents the total number of degrees of freedom of the robot. This space is spanned by the robot's generalized coordinates. The $6 \mathrm{~N}$ dimensional system space hosts the 6 motion components from each of the $N$ rigid body links of the robot. Finally, the 6-dimensional CoM space defines a coordinate frame located at the robot CoM and oriented identical to the inertial reference frame (0). The CoM space is an example of what is more commonly known as the task space ${ }^{5}$.

The Transformation Diagram contains 6 nodes that are arranged in three rows and two columns. Each node corresponds to a vector - a velocity or a momentum - which is denoted in lowercase bold. The three rows correspond,

\footnotetext{
${ }^{4}$ Also called the configuration space.

${ }^{5}$ Also called the operational space [11].
}

\section{Transformation Diagram}

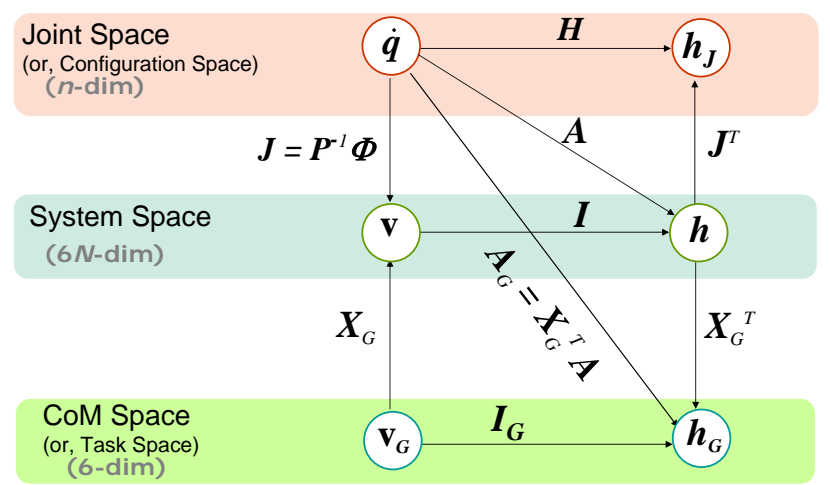

Fig. 3. Transformation Diagram showing the inter-relationships among the velocities and momenta of a robot. These vector quantities can be expressed in joint space, system space, or the CoM space of the robot. The matrices representing the linear transformations between velocities and momenta in different spaces are also shown in this diagram.

from top to bottom, to joint space, system space and CoM space, respectively. The joint space contains $\dot{\boldsymbol{q}}$ and $\boldsymbol{h}_{J}$, the system space contains $\mathbf{v}$ and $\boldsymbol{h}$, and the CoM space contains $\mathbf{v}_{G}$ and $\boldsymbol{h}_{G}$. The left column contains velocity vectors and the right column contains momenta. The mapping between two vectors, shown as a directed line between two nodes, is represented by a matrix, which is denoted in uppercase bold.

The Transformation Diagram contains three types of transformations, which we call horizontal, vertical and diagonal. A horizontal transformation is represented by a square inertia matrix: it occurs within the same space and it maps a velocity vector to a momentum vector. Eq. 16 is a known example of a horizontal transformation within the system space. The mapping from $\dot{\boldsymbol{q}}$ to $\boldsymbol{h}_{J}$, given by

$$
\boldsymbol{h}_{J}=\boldsymbol{H} \dot{\boldsymbol{q}},
$$

is the horizontal transformation within the joint space. $\boldsymbol{H}$ is called the joint-space inertia matrix which is well-known from the standard equations of motion for a robot ${ }^{6}$. The generalized momenta $\boldsymbol{h}_{J}$, which is also called the canonical momenta [20], has not been exploited much for humanoid analysis and control, except in space robotics [16] and collision detection [2].

Within the CoM space, the inertia matrix $\boldsymbol{I}_{G}$ transforms the velocity vector $\mathbf{v}_{G}$ according to

$$
\boldsymbol{h}_{G}=\boldsymbol{I}_{G} \mathbf{v}_{G} \text {. }
$$

${ }^{6}$ The equations of motion for an $n$-dof robot can be expressed as:

$$
\boldsymbol{\tau}=\boldsymbol{H}(\boldsymbol{q}) \ddot{\boldsymbol{q}}+\boldsymbol{C}(\boldsymbol{q}, \dot{\boldsymbol{q}}) \dot{\boldsymbol{q}}+\boldsymbol{\tau}_{g}(\boldsymbol{q}),
$$

where $\boldsymbol{H}$ is the $n \times n$ symmetric, positive-definite joint-space inertia matrix, $\boldsymbol{C}$ is an $n \times n$ matrix such that $\boldsymbol{C} \boldsymbol{q}$ is the vector of Coriolis and centrifugal terms (collectively known as velocity product terms); and $\boldsymbol{\tau}_{g}$ is the vector of gravity terms. 
where $\boldsymbol{h}_{G}$ is the centroidal momentum. $\boldsymbol{I}_{G}$ is called the centroidal composite-rigid-body inertia (CCRBI) matrix [13].

A vertical transformation is represented by a non-square Jacobian or spatial transformation matrix: it relates two velocity or two momentum vectors, but the transformation takes place between two different spaces. The transformation direction for a vertical transformation is not obvious. One direction is typically favored and well-posed, whereas the reverse direction might involve the inversion of a nonsquare matrix with the associated complexity. The vertical transformations corresponding to Eq. 13 and Eq. 24 are shown at the top left and bottom right, respectively, of the Transformation Diagram.

Finally, a diagonal transformation relates two dissimilar vectors between two different spaces. The matrices $\boldsymbol{A}$ (Eq. 21) and $\boldsymbol{A}_{G}$ (Eq. 27) fall in this category. These non-square transformation matrices map $\dot{\boldsymbol{q}}$ to $\boldsymbol{h}$ and $\boldsymbol{h}_{G}$, respectively.

The Transformation Diagram clearly brings out the similarities and differences between $\boldsymbol{H}$ and $\boldsymbol{A}_{G}$. While $\boldsymbol{H}$ is a pure inertia matrix representing a velocity $\rightarrow$ momentum mapping within the same space, the source and destination spaces for $\boldsymbol{A}_{G}$ are different. $\boldsymbol{H}$ and $\boldsymbol{A}_{G}$ are both directly related to the system momentum matrix $\boldsymbol{A}$ and the relationships can be obtained from the Transformation Diagram:

$$
\boldsymbol{H}=\boldsymbol{J}^{T} \boldsymbol{A}
$$

and

$$
\boldsymbol{A}_{G}=\mathbf{X}_{G}^{T} \boldsymbol{A} .
$$

\section{Further Exploration with Transformation Diagram}

The Transformation Diagram contains two vertical transformations (at top right and bottom left) that were not encountered before. The corresponding equations are

$$
\boldsymbol{h}_{J}=\boldsymbol{J}^{T} \boldsymbol{h}
$$

and

$$
\mathbf{v}=\mathbf{X}_{G} \mathbf{v}_{G} .
$$

In order to derive Eq. 34 we will assume that kinetic energy is conserved between the joint space and the system space. In these two spaces, kinetic energy is expressed as:

$$
\begin{aligned}
& K E_{J}=\frac{1}{2} \dot{\boldsymbol{q}}^{T} \boldsymbol{H} \dot{\boldsymbol{q}} \quad \text { and } \\
& K E_{S}=\frac{1}{2} \mathbf{v}^{T} \boldsymbol{I} \mathbf{v} .
\end{aligned}
$$

Assuming $K E_{J}=K E_{S}$ and using Eqs. 29 and 16 we get

$$
\dot{\boldsymbol{q}}^{T} \boldsymbol{h}_{J}=\dot{\boldsymbol{q}}^{T} \boldsymbol{J}^{T} \boldsymbol{h}
$$

in which we have also made use of the fact that $\mathbf{v}=\boldsymbol{J} \dot{\boldsymbol{q}}$ (Eq. 13). If Eq. 38 is to be satisfied for all values of $\dot{\boldsymbol{q}}$, Eq. 34 must also be satisfied. ${ }^{7}$

\footnotetext{
${ }^{7}$ There is an indirect, but more rigorous, way of attaining this equation which involves accelerations and forces. In a redundant system kinetic energy is not conserved between two spaces, in general. A redundant manipulator may have a large joint-space kinetic energy but zero task-space kinetic energy.
}

It is revealing to identify the two primary ways in which $\dot{\boldsymbol{q}}$ can be mapped into $\boldsymbol{h}_{J}$. The direct route $\dot{\boldsymbol{q}} \rightarrow \boldsymbol{h}_{J}$ is given in Eq. 29. A second route progresses counterclockwise as follows: $\dot{\boldsymbol{q}} \rightarrow \mathbf{v} \rightarrow \boldsymbol{h} \rightarrow \boldsymbol{h}_{J}$ and is given by $\boldsymbol{h}_{J}=\boldsymbol{J}^{T} \boldsymbol{I} \boldsymbol{J} \dot{\boldsymbol{q}}$. On equating the two routes, we can obtain the important relationship:

$$
\boldsymbol{H}=\boldsymbol{J}^{T} \boldsymbol{I} \boldsymbol{J} .
$$

In the same way, by traveling from $\mathbf{v}_{G}$ to $\boldsymbol{h}_{G}$ in two different ways: $\boldsymbol{h}_{G}=\boldsymbol{I}_{G} \mathbf{v}_{G}$ and $\boldsymbol{h}_{G}=\mathbf{X}_{G}^{T} \boldsymbol{I} \mathbf{X}_{G} \mathbf{v}_{G}$, we can establish another useful relationship

$$
\boldsymbol{I}_{G}=\mathbf{X}_{G}^{T} \boldsymbol{I} \mathbf{X}_{G}
$$

Although the above equation is a mathematically straightforward congruent transformation, the nature of $\mathbf{X}_{G}$ has not been fully described yet. So far we have assumed the existence of a CoM coordinate "frame" with its origin at the instantaneous robot CoM and its axes orientation identical to that of the inertial frame. Although this description is sufficient to characterize the transformation $\mathrm{X}_{G}^{T}: \boldsymbol{h} \rightarrow \boldsymbol{h}_{G}$ given on the bottom right leg of the Transformation Diagram, the transformation of the bottom left leg, however, is not fully understood. In particular, what system space velocities are obtained through the transformation $\mathbf{X}_{G}: \mathbf{v}_{G} \rightarrow \mathbf{v}$ ? This is what we will discuss in the next section.

\section{Rotational velocity of the CoM "Frame"}

The CoM space, depicted in the third row of the Transformation Diagram, is a specific example of what is more generally known as the task space. For a manipulator robot the frame associated with the task space is rigidly attached to the end effector. The CoM frame, on the other hand, is purely computational and often floats in space. More importantly, although the location of the CoM frame origin can be uniquely computed, there is no physical meaning to its orientation. We had earlier assumed that the CoM frame is oriented identical to the inertial frame. Aside from the fact that our particular choice simplifies the calculations, the choice is nevertheless adhoc and without any known physical grounding. Regardless of the lack of a physically meaningful rotation, the CoM frame is a justifiable frame for our use, mainly due to the importance of its location, with direct significance in balance [7], [22].

Once we know the location of the CoM, we can uniquely compute $\mathbf{X}_{G}^{T}$ as described before. Next, we can compute the CCRBI (see Eq. 31) using Eq. 40. Then, the CoM velocity $\mathbf{v}_{G}$ corresponding to the computed $\boldsymbol{I}_{G}$ is obtained through the relationship

$$
\mathbf{v}_{G}=\boldsymbol{I}_{G}^{-1} \boldsymbol{h}_{G},
$$

where the linear portion of $\mathbf{v}_{G}$ corresponds to the linear CoM velocity. The angular portion $\boldsymbol{\omega}_{G}$ of $\mathbf{v}_{G}$ is then the instantaneous rotational velocity that the CoM frame is to have for a rigid body of instantaneous inertia $\boldsymbol{I}_{G}$ to possess the centroidal momentum $\boldsymbol{h}_{G}$. $\boldsymbol{\omega}_{G}$ can be thought of as an "average velocity" of the humanoid. Another approach to 
defining an "average velocity" of multibody systems can be found in [26].

What is the nature of the transformation $\mathbf{v}=\mathbf{X}_{G} \mathbf{v}_{G}$ then? The only non-trivial solution is the case where all of the links are moving with the same spatial velocity when projected to a common point, which in this case is $G$. If the links are all moving with the same velocity, one way of doing this is to fix them relative to each other. That is, all joint velocities are set to zero except for the floating base joint. In this case, $\boldsymbol{I}_{G}$ is simply the CCRBI.

We note that a computation of the fictitious centroidal angular velocity from inverse inertia and centroidal angular momentum was previously suggested in [15] and [22].

\section{Centroidal Momentum Ellipsoid}

The Centroidal Momentum Matrix maps the robot joint rates to the centroidal momentum. Following the popular concept of the manipulability ellipsoid [25], which quantifies the generation of task space velocities, we can also propose the centroidal momentum ellipsoid. Similar to the manipulability index, a compact quantitative measure of a robot's ability to generate centroidal momentum can then be given by the volume of the ellipsoid [17] as:

$$
w \triangleq \sqrt{\operatorname{det}\left\{\boldsymbol{A}_{G}(\boldsymbol{q}) \boldsymbol{A}_{G}^{T}(\boldsymbol{q})\right\}}
$$

Assuming a singular value representation of $\boldsymbol{A}_{G}(\boldsymbol{q})$ of the standard form, $\boldsymbol{A}_{G}(\boldsymbol{q})=\boldsymbol{U} \Sigma \boldsymbol{V}^{T}$, one can show that

$$
w=\sqrt{\operatorname{det}\left(\Sigma \Sigma^{T}\right)}=\sigma_{1} \sigma_{2} \sigma_{3} \ldots \sigma_{6}
$$

where the $\sigma_{i}$-s are the singular values of $\boldsymbol{A}_{G}(\boldsymbol{q})$. It is well known that while $\dot{\boldsymbol{q}}$ satisfies $\|\dot{\boldsymbol{q}}\|=1, \boldsymbol{h}_{G}$ takes values on a hyper-ellipsoid. The principal axes of the ellipsoid are $\sigma_{i} \boldsymbol{u}_{i}$ for $i=1, \ldots 6$, where $\boldsymbol{u}_{i}$ is the $i^{t h}$ column vector of the orthogonal matrix $\boldsymbol{U}$. A study of the ellipsoid may provide information on the robot's momentum limits along different directions as well as the best means to achieve them.

Due to visualization issues and scale disparities it is customary to construct two different ellipsoids corresponding to the translational and rotational portions of the matrix. We have also separated the linear and angular momentum portions of the Centroidal Momentum Matrix. Consequently, the corresponding ellipsoids separately represent the robot's ability to generate linear and angular momenta at different configurations. Each portion results in 3 singular values.

Our simulation efforts, such as shown in Fig. 4, are ongoing. In Fig. 5 we present the evolution of the three singular values of the linear momentum ellipsoid for the walking motion of a robot. It is remarkable that magnitudes of the singular values are so close.

The centroidal momentum ellipsoid can find use in a number of applications. As an example, it may be valuable to look at the ellipsoid while a humanoid is raising or lowering its body or extending its arms, to determine its ability to regulate its movement with actuators that have speed limitations.

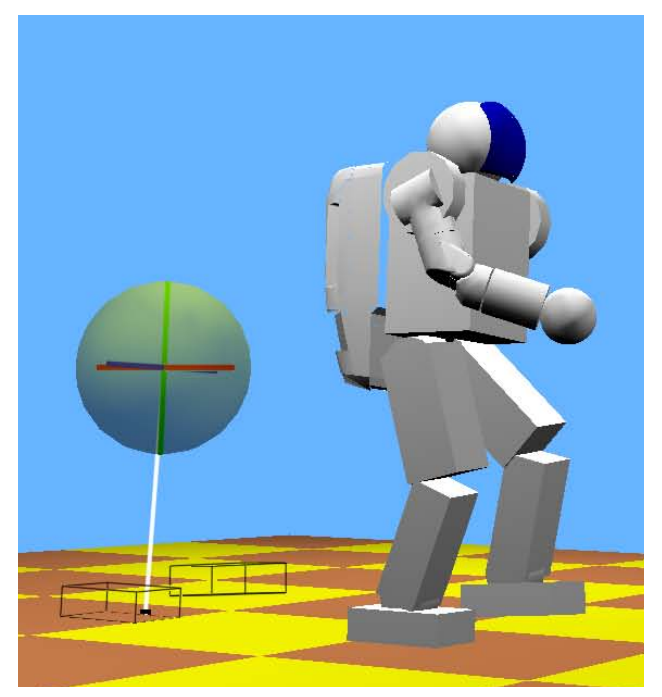

Fig. 4. Centroidal Momentum Matrix ellipsoid shown for a humanoid during a walking motion.

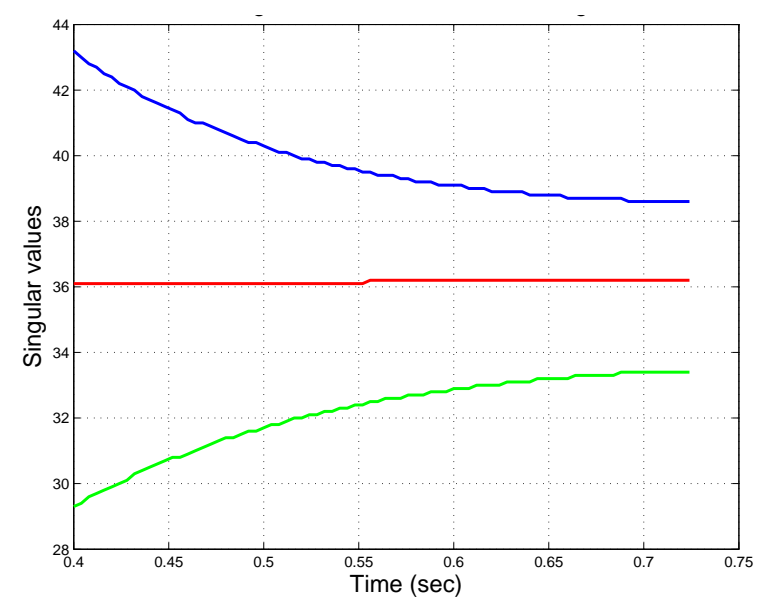

Fig. 5. Evolution of three singular values of the centroidal momentum matrix (only the linear portion) during the walking motion of a humanoid.

\section{CONCLUSIONS AND FUTURE WORK}

In this paper we have derived the expressions for the centroidal momentum of a humanoid robot using spatial notation. The Centroidal Momentum Matrix is the local linear function that maps the robot joint rates to the centroidal momentum. We have demonstrated that this matrix is the product of an inertia and a Jacobian matrix.

We developed the Transformation Diagram that graphically captures the relationships between the velocity and momenta variables of a robot - in joint space, CoM space, as well as the system space. The Transformation Diagram is also helpful in identifying relationships between the transformation matrices.

Next we introduced the centroidal momentum ellipsoid which, similar to the manipulability ellipsoid of a robot, can 
quantitatively indicate the ability of a robot to generate centroidal momentum in a certain direction. We demonstrated this in one simulation example of a humanoid during a walking motion.

For a deeper understanding of the momentum properties of a robot and for practical application, several questions need to be addressed in the future. We will develop an efficient computational algorithm to calculate the Centroidal Momentum Matrix. A number of poorly understood quantities and mapping directions were revealed during the construction of the Transformation Diagram that need to be explored. Finally, analysis of the centroidal momentum ellipsoid will be explored to characterize the momentum capability of a humanoid robot with different configurations of the arms and legs during a variety of movements.

\section{REFERENCES}

[1] M. Abdallah and A. Goswami, "A biomechanically motivated two-phase strategy for biped robot upright balance control," in Proc. of IEEE International Conference on Robotics and Automation (ICRA), Barcelona, Spain, pp. 3707-3713, 2005.

[2] A. De Luca, A. Albu-Schaffer, S. Haddadin, and G. Hirzinger, "Collision detection and safe reaction with the DLR-III lightweight manipulator arm", in Proc. of IEEE/RSJ International Conference on Intelligent Robots and Systems (IROS), Beijing, China, pp. 1623-1630, 2006.

[3] A. C. Fang and N. Pollard, "Efficient synthesis of physically valid human motion", in ACM Transactions on Graphics, ACM SIGGRAPH Proceedings, vol. 22, no. 3, pp. 417-426, 2003.

[4] R. Featherstone, Rigid Body Dynamics Algorithms, New York: Springer, 2008.

[5] R. Featherstone and D. E. Orin, "Robot dynamics: equations and algorithms," in Proc. of IEEE International Conference on Robotics and Automation, San Francisco, pp. 826-834, April 2000.

[6] R. Featherstone and D. E. Orin, "Dynamics," Chapter 2 in Springer Handbook of Robotics, B. Siciliano and O. Khatib, Eds., New York: Springer, 2008.

[7] A. Goswami and V. Kallem, "Rate of change of angular momentum and balance maintenance of biped robots," in Proc. of IEEE International Conference on Robotics and Automation (ICRA), New Orleans, LA, 2004.

[8] H. Herr and M. B. Popovic, "Angular momentum in human walking," The Journal of Experimental Biology, vol. 211, pp. $467-481,2008$.

[9] A. G. Hofmann, Robust Execution of Bipedal Walking Tasks from Biomechanical Principles, Ph.D. Thesis, MIT, 2005.

[10] S. Kajita, F. Kanehiro, K. Kaneko, K. Fujiwara, K. Harada, $\mathrm{K}$. Yokoi, and H. Hirukawa, "Resolved momentum control: humanoid motion planning based on the linear and angular momentum", in Proc. of IEEE/RSJ International Conference on Intelligent Robots and Systems (IROS), Las Vegas, NV, USA, pp. 1644-1650, 2003.

[11] O. Khatib, "A unified approach to motion and force control of robot manipulators: the operational space formulation," IEEE Transactions on Robotics and Automation, vol. 3, no. 1, pp. 43-53, 1987.

[12] T. Komura, H. Leung, S. Kudoh, and J. Kuffner, "A feedback controller for biped humanoids that can counteract large perturbations during gait," in Proc. of IEEE International Conference on Robotics and Automation (ICRA), Barcelona, Spain, pp. 2001-2007, 2005.
[13] S.-H. Lee and A. Goswami, "Reaction Mass Pendulum (RMP): an explicit model for centroidal angular momentum of humanoid robots," in Proc. of IEEE International Conference on Robotics and Automation, Rome, Italy, April 2007.

[14] K. Mitobe, G. Capi, and Y. Nasu, "A new control method for walking robots based on angular momentum", Mechatronics, vol.14, pp. 163-174, 2004.

[15] Y. Morita and K. Ohnishi, "Attitude control of hopping robot using angular momentum", in Proc. of IEEE International Conference on Industrial Technology, vol. 1, pp. 173-178, 2003.

[16] D. Nenchev, Y. Umetani, and K. Yoshida, "Analysis of a redundant free-flying spacecraft/manipulator system", IEEE Transactions on Robotics and Automation, vol. 8, no. 1, pp. 16, 1992.

[17] Y. Nakamura, Advanced Robotics: Redundancy and Optimization, Addision-Wesley Publishing Company, Reading, MA, USA, 1991

[18] N. Naksuk, Y. Mei, and C. S. George Lee, "Humanoid trajectory generation: an iterative approach based on movement and angular momentum criteria", in Proc. of the IEEE-RAS/RSJ International Conference on Humanoid Robots, Santa Monica, CA, October 2004.

[19] N. Naksuk, C. S. George Lee, and S. Rietdyk, "Wholebody human to humanoid motion transfer", in Proc. of IEEE IEEE/RSJ International Conference on Intelligent Robots and Systems (IROS), Edmonton, Alberta, Canada, pp. 104-109, 2005.

[20] J. Naudet, Forward Dynamics of Multibody Systems: A Recursive Hamiltonian Approach, $\mathrm{PhD}$ Thesis, Vrije Universiteit, Brussels, Belgium, September 2005.

[21] K. Nishiwaki, S. Kagami, Y. Kuniyoshi, M. Inaba, and H. Inoue, "Online generation of humanoid walking motion based on a fast generation method of motion pattern that follows desired ZMP," in Proc. of the IEEE/RSJ International Conference on Intelligent Robots and Systems (IROS), pp. 2684-2689, 2002.

[22] M. B. Popovic, A. Hofmann, and H. Herr, "Zero spin angular momentum control: definition and applicability," in Proc. of the IEEE-RAS/RSJ International Conference on Humanoid Robots, Santa Monica, CA, 2004.

[23] R. E. Roberson and R. Schwertassek, Dynamics of Multibody Systems, Berlin/Heidelberg/New York: Springer-Verlag, 1988.

[24] G. Rodriguez, A. Jain, and K. Kreutz-Delgado, "A spatial operator algebra for manipulator modelling and control," International Journal of Robotics Research, vol. 10, no. 4, pp. 371-381, 1991.

[25] T. Yoshikawa, Foundations of Robotics: Analysis and Control, The MIT Press, 1990.

[26] H. Essén, "Average Angular Velocity," European Journal of Physics, vol. 14, pp. 201-205, 1993. 